\title{
Asymptotic Behavior for a Viscoelastic Wave Equation with a Time-varying Delay Term
}

\section{WU Shun Tang *}

General Education Center, National Taipei University of Technology, Taipei, Taiwan 106.

Received 30 August 2015; Accepted 28 February 2016

Abstract. The following viscoelastic wave equation with a time-varying delay term in internal feedback

$$
\left|u_{t}\right|^{\rho} u_{t t}-\Delta u-\Delta u_{t t}+\int_{0}^{t} g(t-s) \Delta u(s) \mathrm{d} s+\mu_{1} u_{t}(x, t)+\mu_{2} u_{t}(x, t-\tau(t))=0,
$$

is considered in a bounded domain. Under appropriate conditions on $\mu_{1}, \mu_{2}$ and on the kernel $g$, we establish the general decay result for the energy by suitable Lyapunov functionals.

AMS Subject Classifications: 35L05, 35L15, 35L70, 93D15

Chinese Library Classifications: O175.27

Key Words: Global existence; asymptotic behavior; general decay; time-varying delay.

\section{Introduction}

In this paper, we consider the initial boundary value problem for a nonlinear viscoelastic equation with a linear damping and a time-varying delay term of the form:

$$
\begin{aligned}
& \left|u_{t}\right|^{\rho} u_{t t}-\Delta u-\Delta u_{t t}+\int_{0}^{t} g(t-s) \Delta u(s) \mathrm{d} s+\mu_{1} u_{t}(x, t) \\
& \quad+\mu_{2} u_{t}(x, t-\tau(t))=0, \quad \text { in } \Omega \times(0, \infty), \\
& u_{t}(x, t)=f_{0}(x, t), \quad x \in \Omega, t \in[-\tau(0), 0), \\
& u(x, 0)=u_{0}(x), u_{t}(x, 0)=u_{1}(x), \quad x \in \Omega, \\
& u(x, t)=0, \quad x \in \partial \Omega, t \geq 0,
\end{aligned}
$$

${ }^{*}$ Corresponding author. Email address: stwu@ntut.edu.tw (S. T. Wu) 
where $\rho>0, \Omega \subset R^{N}(N \geq 1)$ is a bounded domain with a smooth boundary $\partial \Omega$. Moreover, $\mu_{1}$ and $\mu_{2}$ are real constants with $\mu_{1}>0, \tau(t)>0$ represents the time-varying delay, $g$ is the kernel of the memory term and the initial data $\left(u_{0}, u_{1}, f_{0}\right)$ are given functions belonging to suitable spaces.

It is well known that delay effects, which arise in many practical problems, might induce some instabilities, see [1-6]. Hence, questions related to the behavior of solutions for the PDEs with time delay effects have become active area of research in recent years. Many authors have focused on this problem and several results concerning existence, decay and instability have been obtained, see [2-12] and reference therein. In this regard, Datko et al. [4] showed that a small delay in a boundary control is a source of instability. Nicaise et al. [7] studied a system of wave equation with a linear boundary damping term with a delay as follows

$$
\begin{cases}u_{t t}-\Delta u=0, & \text { in } \Omega \times(0, \infty), \\ u(x, t)=0, & x \in \Gamma_{0}, t \geq 0, \\ \frac{\partial}{\partial v}(x, t)=\mu_{1} u_{t}(x, t)+\mu_{2} u_{t}(x, t-\tau), & \text { in } \Gamma_{1} \times(0, \infty), \\ u(x, 0)=u_{0}(x), u_{t}(x, 0)=u_{1}(x), & x \in \Omega, \\ u_{t}(x, t-\tau)=f_{0}(x, t-\tau), & x \in \Omega, t \in(0, \tau) .\end{cases}
$$

where $v$ is the unit outward normal to $\partial \Omega$. Under the condition

$$
\mu_{2}<\mu_{1}
$$

they established a stabilization result by applying inequalities obtained from Carleman estimates for the wave equation by Lasiecka et al. [13] and by using compactness-uniqueness arguments. Conversely, if (1.6) does not hold, they showed that there exists a sequence of delays for which the corresponding solution of (1.5) is unstable. And, they also obtained the same results if both the damping and the delay act in the domain.

The case of time-varying delay in the wave equation has been studied by Nicaise et al. [10] in one space dimension, in which they obtained an exponential decay result subject to the condition

$$
\mu_{2} \leq \sqrt{1-d} \mu_{1}
$$

where $d$ is a constant such that

$$
\tau^{\prime}(t) \leq d<1, \quad \forall t>0 .
$$

Later, under the condition $\left|\mu_{2}\right|<\sqrt{1-d} \mu_{1}$ in which the positivity of the coefficient $\mu_{2}$ is not necessary, Nicaise et al. [11] extended this result to general space dimension. In fact, they proved exponential stability of the solution for the wave equation with a timevarying delay in the boundary condition in a bounded and smooth domain in $R^{N}$. Recently, inspired the works of Nicaise et al. [11] and M. Kirane et al. [5], Liu [14] considered 
the following problem

$$
u_{t t}-\Delta u+\int_{0}^{t} g(t-s) \Delta u(s) \mathrm{d} s+\mu_{1} u_{t}(x, t)+\mu_{2} u_{t}(x, t-\tau(t))=0,
$$

in a bounded domain with the conditions (1.2)-(1.4). In that work, they established general decay results of the energy via suitable Lyapunov functionals under the condition $\left|\mu_{2}\right|<\sqrt{1-d} \mu_{1}$.

In the absence of the delay term (i.e. $\left.\mu_{2}=0\right)$ in (1.1), related problems have been extensively studied and there are numerous results related to existence, asymptotic behavior and blow-up of solutions. For example, Cavalcanti et al. [15] considered the following problem:

$$
\left|u_{t}\right|^{\rho} u_{t t}-\Delta u-\Delta u_{t t}+\int_{0}^{t} g(t-s) \Delta u(s) \mathrm{d} s-\gamma \Delta u_{t}=0,
$$

with the same initial and boundary conditions (1.3)-(1.4), where a global existence result for $\gamma \geq 0$ and an exponential decay result for $\gamma>0$ were established under the assumptions $0<\rho \leq \frac{2}{N-2}$ if $N \geq 3$ or $\rho>0$ if $N=1,2$ and $g(t)$ decays exponentially. Lately, these decay results were extended by Messaoudi and Tatar [16] to a situation where a source term is present. Recently, Messaoudi and Tatar [17] studied problem (1.10) for case of $\gamma=0$, they showed that the solution goes to zero with an exponential or polynomial rate under some restrictions on the relaxation function. For other related works, we refer the readers to [18-23] and references therein.

Motivated by previous works, it is interesting to investigate whether there are similar decay results as in [14] for problem (1.1)-(1.4), in which more general form than that of problem (1.9) is considered. Our proof technique closely follows the arguments of $[14,23]$, with the modifications being needed for our problem. The content of this paper is organized as follows. In Section 2, we provide assumptions that will be used later, state and prove the existence result Theorem 2.3. In Section 3, we prove our stability result that is given in Theorem 3.5. Finally, we give some examples to illustrate our result.

\section{Preliminary results}

In this section, we shall give some assumptions which will be used throughout this work. We use the standard Lebesgue space $L^{p}(\Omega)$ and Sobolev space $H_{0}^{1}(\Omega)$ with their usual products and norms.

Lemma 2.1 (Sobolev-Poincaré inequality). Let $2 \leq p \leq \frac{2 N}{N-2}$, the inequality

$$
\|u\|_{p} \leq c_{s}\|\nabla u\|_{2}, \quad \text { for } u \in H_{0}^{1}(\Omega) \text {, }
$$

holds with some positive constant $c_{s}$. 
Assume that $\rho$ satisfies

$$
0<\rho \leq \frac{2}{N-2} \text { if } N \geq 3 \text { or } \rho>0 \text { if } N=1,2 .
$$

Regarding the relaxation function $g(t)$, we assume that it verifies:

(A1) $g: R^{+} \rightarrow R^{+}$is a bounded $C^{1}$ function satisfying

$$
1-\int_{0}^{\infty} g(s) \mathrm{d} s=l>0,
$$

and there exists a positive nonincreasing function $\xi$ such that, for $t \geq 0$,

$$
g^{\prime}(t) \leq-\xi(t) g(t) \quad \text { and } \quad \int_{0}^{\infty} \xi(s) \mathrm{d} s=\infty .
$$

(A2) For the time-varying delay $\tau(t)$, we assume as in [24] that there exist positive constants $\tau_{0}$ and $\tau_{1}$ such that

$$
0<\tau_{0} \leq \tau(t) \leq \tau_{1}, \quad t>0 .
$$

Moreover, we assume that $\tau \in W^{2, \infty}[0, T], T>0$ and

$$
\tau^{\prime}(t) \leq d<1, \quad t>0,
$$

and that $\mu_{1}, \mu_{2}$ satisfy

$$
\left|\mu_{2}\right|<\sqrt{1-d} \mu_{1} .
$$

In order to prove the existence of solutions of problem (1.1)-(1.4), we introduced the new variable $z$ as in [24],

$$
z(x, \kappa, t)=u_{t}(x, t-\tau(t) \kappa), \quad x \in \Omega, \kappa \in(0,1),
$$

which implies that

$$
\tau(t) z_{t}(x, \kappa, t)+\left(1-z^{\prime}(t) \kappa\right) z_{\kappa}(x, \kappa, t)=0, \quad \text { in } \Omega \times(0,1) \times(0, \infty) .
$$

Therefore, problem (1.1)-(1.4) can be transformed as follows

$$
\begin{cases}\left|u_{t}\right|^{\rho} u_{t t}-\Delta u-\Delta u_{t t}+\int_{0}^{t} g(t-s) \Delta u(s) \mathrm{d} s+\mu_{1} u_{t}(x, t) \\ \quad+\mu_{2} z(x, 1, t)=0, \quad \text { in } \Omega \times(0, \infty), & \\ \tau(t) z_{t}(x, \kappa, t)+\left(1-z^{\prime}(t) \kappa\right) z_{\kappa}(x, \kappa, t)=0, & x \in \Omega, \kappa \in(0,1), t>0, \\ z(x, 0, t)=u_{t}(x, t), & x \in \Omega, t>0, \\ z(x, \kappa, 0)=f_{0}(x,-\tau(0) \kappa), & x \in \Omega, \\ u(x, 0)=u_{0}(x), u_{t}(x, 0)=u_{1}(x), & x \in \Omega, \\ u(x, t)=0, & x \in \partial \Omega, t \geq 0,\end{cases}
$$


Now, we are ready to give the well-posedness of problem (1.1)-(1.4) which can be obtained by the arguments of $[5,23]$.

Theorem 2.2. Suppose that (2.1), and (A1)-(A2) hold. Assume further that $u_{0}, u_{1} \in H_{0}^{1}(\Omega)$ and $f_{0} \in L^{2}(\Omega \times(0,1))$. Then there exists a unique solution $(u, z)$ of $(2.7)$ satisfying

$$
u, u_{t} \in C\left([0, T) ; H_{0}^{1}(\Omega)\right) \text { and } z \in C\left([0, T) ; L^{2}(\Omega \times(0,1))\right),
$$

for $T>0$.

\section{Asymptotic behavior}

In this section, we shall investigate the asymptotic behavior of problem (1.1)-(1.4). Now, inspired by [24], we define the energy function of problem (1.1)-(1.4) as

$$
\begin{gathered}
E(t)=\frac{1}{\rho+2}\left\|u_{t}\right\|_{\rho+2}^{\rho+2}+\frac{1}{2}\left(1-\int_{0}^{t} g(s) \mathrm{d} s\right)\|\nabla u(t)\|_{2}^{2}+\frac{1}{2}(g \circ \nabla u)(t) \\
+\frac{1}{2}\left\|\nabla u_{t}(t)\right\|_{2}^{2}+\frac{\zeta}{2} \int_{t-\tau(t)}^{t} \int_{\Omega} e^{\lambda(s-t)} u_{t}^{2}(x, s) \mathrm{d} x \mathrm{~d} s
\end{gathered}
$$

where

$$
(g \circ \phi)(t)=\int_{0}^{t} g(t-s) \int_{\Omega}|\phi(s)-\phi(t)|^{2} \mathrm{~d} x \mathrm{~d} s
$$

and $\zeta$ is a positive constant such that

$$
2 \mu_{1}-\frac{\left|\mu_{2}\right|}{\sqrt{1-d}}-\zeta>0, \quad \zeta-\frac{\left|\mu_{2}\right|}{\sqrt{1-d}}>0,
$$

and

$$
\lambda<\frac{1}{\tau_{1}}\left|\log \frac{\left|\mu_{2}\right|}{\zeta \sqrt{1-d}}\right| .
$$

Lemma 3.1. $E(t)$ is a nonincreasing function on $[0, T]$ and

$$
\begin{aligned}
E^{\prime}(t)=-c_{1}\left\|u_{t}\right\|_{2}^{2}-c_{2} \int_{\Omega} u_{t}^{2}(x, t-\tau(t)) \mathrm{d} x+\frac{1}{2}\left(g^{\prime} \circ \nabla u\right)(t)-\frac{1}{2} g(t)\|\nabla u(t)\|_{2}^{2} \\
\quad-\frac{\lambda \zeta}{2} \int_{t-\tau(t)}^{t} \int_{\Omega} e^{-\lambda(t-s)} u_{t}^{2}(x, s) \mathrm{d} x \mathrm{~d} s \\
\leq \frac{1}{2}\left(g^{\prime} \circ \nabla u\right)(t)-\frac{1}{2} g(t)\|\nabla u(t)\|_{2}^{2} \leq 0, \quad \forall t \geq 0,
\end{aligned}
$$

where

$$
c_{1}=\mu_{1}-\frac{\left|\mu_{2}\right|}{2 \sqrt{1-d}}-\frac{\zeta}{2}>0 \text { and } c_{2}=\frac{\zeta}{2}(1-d) e^{-\lambda \tau_{1}}-\frac{\sqrt{1-d}\left|\mu_{2}\right|}{2}>0 .
$$


Proof. Differentiating (3.1) and using (1.1), integrating by parts over $\Omega$ and exploiting the assumption (A1), (2.4)-(2.6) and (3.2)-(3.3), we obtain

$$
\begin{aligned}
E^{\prime}(t) \leq-c_{1}\left\|u_{t}\right\|_{2}^{2}-c_{2} \int_{\Omega} u_{t}^{2}(x, t-\tau(t)) \mathrm{d} x+\frac{1}{2}\left(g^{\prime} \circ \nabla u\right)(t)-\frac{1}{2} g(t)\|\nabla u(t)\|_{2}^{2} \\
\quad-\frac{\lambda \zeta}{2} \int_{t-\tau(t)}^{t} \int_{\Omega} e^{-\lambda(t-s)} u_{t}^{2}(x, s) \mathrm{d} x \mathrm{~d} s \\
\leq \frac{1}{2}\left(g^{\prime} \circ \nabla u\right)(t)-\frac{1}{2} g(t)\|\nabla u(t)\|_{2}^{2} \leq 0, \quad \forall t \geq 0 .
\end{aligned}
$$

Remark 3.2. It follows from the definition of $E(t)$ that the energy function is uniformly bounded and decreasing in $t$, which implies that

$$
l\|\nabla u\|_{2}^{2}+\left\|\nabla u_{t}\right\|_{2}^{2} \leq 2 E(t) \leq 2 E(0), \quad \forall t \geq 0 .
$$

Now, we define

$$
G(t)=M E(t)+\varepsilon \Phi(t)+\Psi(t),
$$

where $M$ and $\varepsilon$ are positive constants which will be specified later and

$$
\begin{aligned}
& \Phi(t)=\frac{1}{\rho+1} \int_{\Omega}\left|u_{t}\right|^{\rho} u_{t} u \mathrm{~d} x+\int_{\Omega} \nabla u_{t}(t) \cdot \nabla u(t) \mathrm{d} x, \\
& \Psi(t)=\int_{\Omega}\left(\Delta u_{t}-\frac{1}{\rho+1}\left|u_{t}\right|^{\rho} u_{t}\right) \int_{0}^{t} g(t-s)(u(t)-u(s)) \mathrm{d} s \mathrm{~d} x .
\end{aligned}
$$

The following lemma tells us that $G(t)$ and $E(t)$ are equivalent.

Lemma 3.3. Let $u$ be a solution of problem (1.1)-(1.4), then there exist two positive constants $\beta_{1}$ and $\beta_{2}$ such that

$$
\beta_{1} E(t) \leq G(t) \leq \beta_{2} E(t), \quad \forall t \geq 0,
$$

for M sufficiently large.

Proof. Using Young's inequality, Lemma 2.1 and (3.4), we have

$$
\begin{aligned}
\left.\left|\frac{1}{\rho+1} \int_{\Omega}\right| u_{t}\right|^{\rho} u_{t} u \mathrm{~d} x \mid \leq \frac{1}{\rho+2} & \left\|u_{t}\right\|_{\rho+2}^{\rho+2} \\
& +\frac{c_{s}^{\rho+2}}{(\rho+2)(\rho+1)}\left(\frac{2 E(0)}{l}\right)^{\frac{\rho}{2}}\|\nabla u\|_{2}^{2}
\end{aligned}
$$

and

$$
\left|\int_{\Omega} \nabla u_{t}(t) \cdot \nabla u(t) \mathrm{d} x\right| \leq \frac{1}{2}\left\|\nabla u_{t}\right\|_{2}^{2}+\frac{1}{2}\|\nabla u\|_{2}^{2}
$$


Further, we observe from (3.7) that

$$
\begin{array}{r}
\Psi(t)=-\int_{\Omega} \nabla u_{t} \cdot \int_{0}^{t} g(t-s)(\nabla u(t)-\nabla u(s)) \mathrm{d} s \mathrm{~d} x \\
-\frac{1}{\rho+1} \int_{\Omega}\left|u_{t}\right|^{\rho} u_{t} \int_{0}^{t} g(t-s)(u(t)-u(s)) \mathrm{d} s \mathrm{~d} x .
\end{array}
$$

By Young's inequality, Hölder's inequality (2.2) and (3.4), we have

$$
\begin{aligned}
& \left|-\int_{\Omega} \nabla u_{t} \cdot \int_{0}^{t} g(t-s)(\nabla u(t)-\nabla u(s)) \mathrm{d} s \mathrm{~d} x\right| \\
\leq & \frac{1}{2}\left\|\nabla u_{t}\right\|_{2}^{2}+\frac{1}{2} \int_{\Omega}\left(\int_{0}^{t} g(t-s)(\nabla u(t)-\nabla u(s)) \mathrm{d} s\right)^{2} \mathrm{~d} x \\
\leq & \frac{1}{2}\left\|\nabla u_{t}\right\|_{2}^{2}+\frac{1-l}{2}(g \circ \nabla u)(t),
\end{aligned}
$$

and

$$
\begin{aligned}
& \left.\left|-\frac{1}{\rho+1} \int_{\Omega}\right| u_{t}\right|^{\rho} u_{t} \int_{0}^{t} g(t-s)(u(t)-u(s)) \mathrm{d} s \mathrm{~d} x \mid \\
& \leq \frac{1}{\rho+2}\left(\left\|u_{t}\right\|_{\rho+2}^{\rho+2}+\frac{1}{\rho+1} \int_{\Omega}\left(\int_{0}^{t} g(t-s)(u(t)-u(s)) \mathrm{d} s\right)^{\rho+2} \mathrm{~d} x\right) \\
& \leq \frac{1}{\rho+2}\left(\left\|u_{t}\right\|_{\rho+2}^{\rho+2}+\frac{(1-l)^{\rho+1} c_{s}^{\rho+2}}{\rho+1} \int_{0}^{t} g(t-s)\|\nabla u(t)-\nabla u(s)\|_{2}^{\rho+2} \mathrm{~d} s\right) \\
& \leq \frac{1}{\rho+2}\left(\left\|u_{t}\right\|_{\rho+2}^{\rho+2}+\frac{(1-l)^{\rho+1} c_{s}^{\rho+2}}{\rho+1}\left(\frac{4 E(0)}{l}\right)^{\frac{\rho}{2}}(g \circ \nabla u)(t)\right) .
\end{aligned}
$$

Combining (3.8)-(3.12) with (3.5) yields

$$
\begin{aligned}
& |G(t)-M E(t)|=\varepsilon \Phi(t)+\Psi(t) \\
\leq & c_{3}\left\|u_{t}\right\|_{\rho+2}^{\rho+2}+c_{4}\|\nabla u\|_{2}^{2}+c_{5}\left\|\nabla u_{t}\right\|_{2}^{2}+c_{6}(g \circ \nabla u)(t) \\
\leq & c_{7} E(t),
\end{aligned}
$$

where $c_{3}=\frac{1+\varepsilon}{\rho+2}, c_{4}=\varepsilon\left(\frac{c_{s}^{\rho+2}}{(\rho+2)(\rho+1)}\left(\frac{2 E(0)}{l}\right)^{\frac{\rho}{2}}+\frac{1}{2}\right), c_{5}=\frac{\varepsilon+1}{2}$,

$$
c_{6}=\varepsilon\left(\frac{1-l}{2}+\frac{(1-l)^{\rho+1} c_{s}^{\rho+2}}{(\rho+2)(\rho+1)}\left(\frac{4 E(0)}{l}\right)^{\frac{\rho}{2}}\right), \text { and } c_{7}=\max \left(c_{3}, c_{4}, c_{5}, c_{6}\right) .
$$

Thus, from the definition of $E(t)$ by (3.1) and selecting $M$ sufficiently large, there exist two positive constants $\beta_{1}$ and $\beta_{2}$ such that

$$
\beta_{1} E(t) \leq G(t) \leq \beta_{2} E(t) .
$$


Theorem 3.4. Let $u_{0}, u_{1} \in H_{0}^{1}(\Omega)$ be given. Suppose that (2.1), (A1)-(A2) and (3.2)-(3.3) hold. Then for each $t_{0}>0$ the solution energy of problem (1.1)-(1.4) satisfies

$$
E(t) \leq K e^{-\alpha \int_{t_{0}}^{t} \xi(s) \mathrm{d} s}, \quad t \geq t_{0},
$$

where $\alpha$ and $K$ are some positive constants given in the proof.

Proof. In order to obtain the decay result of $E(t)$, it is sufficient to prove that of $G(t)$. To this end, we need to estimate the derivative of $G(t)$. It follows from (3.6) that

$$
\begin{gathered}
\Phi^{\prime}(t)=-\|\nabla u\|_{2}^{2}+\int_{\Omega} \nabla u(t) \cdot \int_{0}^{t} g(t-s) \nabla u(s) \mathrm{d} s \mathrm{~d} x-\mu_{1} \int_{\Omega} u_{t}(x, t) u(t) \mathrm{d} x \\
-\mu_{2} \int_{\Omega} u_{t}(x, t-\tau(t)) u(t) \mathrm{d} x+\frac{1}{\rho+1}\left\|u_{t}\right\|_{\rho+2}^{\rho+2}+\left\|\nabla u_{t}\right\|_{2}^{2} .
\end{gathered}
$$

Using Hölder's inequality, Young's inequality and (A1), we obtain, for $\eta>0, \delta_{1}>0$,

$$
\begin{aligned}
& \left|\int_{\Omega} \nabla u(t) \cdot \int_{0}^{t} g(t-s) \nabla u(s) \mathrm{d} s \mathrm{~d} x\right| \\
\leq & \frac{1}{2}\|\nabla u\|_{2}^{2}+\frac{1}{2} \int_{\Omega}\left(\int_{0}^{t} g(t-s)(|\nabla u(s)-\nabla u(t)|+|\nabla u(t)|) \mathrm{d} s\right)^{2} \mathrm{~d} x \\
\leq & \frac{1+(1+\eta)(1-l)^{2}}{2}\|\nabla u\|_{2}^{2}+\frac{\left(1+\frac{1}{\eta}\right)(1-l)}{2}(g \circ \nabla u)(t), \\
& \left|\int_{\Omega} u_{t}(x, t) u(t) \mathrm{d} x\right| \leq \delta_{1} c_{s}^{2}\|\nabla u\|_{2}^{2}+\frac{c_{s}^{2}}{4 \delta_{1}}\left\|\nabla u_{t}\right\|_{2}^{2},
\end{aligned}
$$

and

$$
\left|\int_{\Omega} u_{t}(x, t-\tau(t)) u(t) \mathrm{d} x\right| \leq \delta_{1} c_{s}^{2}\|\nabla u\|_{2}^{2}+\frac{1}{4 \delta_{1}} \int_{\Omega} u_{t}^{2}(x, t-\tau(t)) \mathrm{d} x
$$

Letting $\eta=\frac{l}{1-l}$ in (3.15) and employing (3.16)-(3.17), we derive from (3.14) that

$$
\begin{aligned}
& \Phi^{\prime}(t) \leq-(\left.\frac{l}{2}-\delta_{1} c_{s}^{2}\left(\mu_{1}+\left|\mu_{2}\right|\right)\right)\|\nabla u\|_{2}^{2}+\frac{1-l}{2 l}(g \circ \nabla u)(t)+\frac{1}{\rho+1}\left\|u_{t}\right\|_{\rho+2}^{\rho+2} \\
&+\frac{\left|\mu_{2}\right|}{4 \delta_{1}} \int_{\Omega} u_{t}^{2}(x, t-\tau(t)) \mathrm{d} x+\left(\frac{\mu_{1} c_{s}^{2}}{4 \delta_{1}}+1\right)\left\|\nabla u_{t}\right\|_{2}^{2} .
\end{aligned}
$$

Taking the derivative of $\Psi(t)$ in (3.7) and using Eq. (1.1), we get

$$
\begin{aligned}
\Psi^{\prime}(t)=\int_{\Omega} \nabla & u(t) \cdot \int_{0}^{t} g(t-s)(\nabla u(t)-\nabla u(s)) \mathrm{d} s \mathrm{~d} x \\
& -\int_{\Omega}\left(\int_{0}^{t} g(t-s) \nabla u(s) \mathrm{d} s\right) \cdot\left(\int_{0}^{t} g(t-s)(\nabla u(t)-\nabla u(s)) \mathrm{d} s\right) \mathrm{d} x
\end{aligned}
$$




$$
\begin{aligned}
& +\mu_{1} \int_{\Omega} u_{t}(t) \int_{0}^{t} g(t-s)(u(t)-u(s)) \mathrm{d} s \mathrm{~d} x \\
& +\mu_{2} \int_{\Omega} u_{t}(x, t-\tau(t)) \int_{0}^{t} g(t-s)(u(t)-u(s)) \mathrm{d} s \mathrm{~d} x \\
& -\int_{\Omega} \nabla u_{t}(t) \cdot \int_{0}^{t} g^{\prime}(t-s)(\nabla u(t)-\nabla u(s)) \mathrm{d} s \mathrm{~d} x \\
& -\frac{1}{\rho+1} \int_{\Omega}\left|u_{t}\right|^{\rho} u_{t} \int_{0}^{t} g^{\prime}(t-s)(u(t)-u(s)) \mathrm{d} s \mathrm{~d} x \\
& -\left(\int_{0}^{t} g(s) \mathrm{d} s\right)\left\|\nabla u_{t}\right\|_{2}^{2}-\frac{1}{\rho+1}\left(\int_{0}^{t} g(s) \mathrm{d} s\right)\left\|u_{t}\right\|_{\rho+2}^{\rho+2} .
\end{aligned}
$$

In what follows we will estimate the right hand side of (3.19). Using Hölder's inequality, Young's inequality and (2.2), for $\delta>0$, we have

$$
\begin{aligned}
& \left|\int_{\Omega} \nabla u(t) \cdot \int_{0}^{t} g(t-s)(\nabla u(t)-\nabla u(s)) \mathrm{d} s \mathrm{~d} x\right| \\
\leq & \delta\|\nabla u\|_{2}^{2}+\frac{1-l}{4 \delta}(g \circ \nabla u)(t) .
\end{aligned}
$$

and

$$
\begin{aligned}
& \left|\int_{\Omega}\left(\int_{0}^{t} g(t-s) \nabla u(s) \mathrm{d} s\right) \cdot\left(\int_{0}^{t} g(t-s)(\nabla u(t)-\nabla u(s)) \mathrm{d} s\right) \mathrm{d} x\right| \\
\leq & \delta \int_{\Omega}\left(\int_{0}^{t} g(t-s)|\nabla u(s)| \mathrm{d} s\right)^{2} \mathrm{~d} x+\frac{1}{4 \delta} \int_{\Omega}\left(\int_{0}^{t} g(t-s)|\nabla u(t)-\nabla u(s)| \mathrm{d} s\right)^{2} \mathrm{~d} x \\
\leq & 2 \delta(1-l)^{2}\|\nabla u\|_{2}^{2}+\left(2 \delta+\frac{1}{4 \delta}\right)(1-l)(g \circ \nabla u)(t) .
\end{aligned}
$$

Utilizing Young's inequality, Lemma 2.1 and (A1), the third term, the fourth term and the fifth term on the right hand side of (3.19) can be estimated as, for $\delta_{2}>0$,

$$
\begin{aligned}
& \left|\mu_{1} \int_{\Omega} u_{t}(t) \int_{0}^{t} g(t-s)(u(t)-u(s)) \mathrm{d} s \mathrm{~d} x\right| \\
\leq & \delta_{2} \mu_{1} c_{s}^{2}\left\|\nabla u_{t}\right\|_{2}^{2}+\frac{\mu_{1} c_{s}^{2}(1-l)}{4 \delta_{2}}(g \circ \nabla u)(t), \\
& \left|\mu_{2} \int_{\Omega} u_{t}(x, t-\tau(t)) \int_{0}^{t} g(t-s)(u(t)-u(s)) \mathrm{d} s \mathrm{~d} x\right| \\
\leq & \left|\mu_{2}\right| \delta_{2} \int_{\Omega} u_{t}^{2}(x, t-\tau(t)) \mathrm{d} x+\frac{\left|\mu_{2}\right|(1-l) c_{s}^{2}}{4 \delta_{2}}(g \circ \nabla u)(t),
\end{aligned}
$$

and

$$
\left|\int_{\Omega} \nabla u_{t}(t) \int_{0}^{t} g^{\prime}(t-s)(\nabla u(t)-\nabla u(s)) \mathrm{d} s \mathrm{~d} x\right|
$$




$$
\begin{aligned}
& \leq \delta_{2}\left\|\nabla u_{t}\right\|_{2}^{2}+\frac{1}{4 \delta_{2}} \int_{\Omega}\left(\int_{0}^{t} g^{\prime}(t-s)(\nabla u(t)-\nabla u(s)) \mathrm{d} s\right)^{2} \mathrm{~d} x \\
& \leq \delta_{2}\left\|\nabla u_{t}\right\|_{2}^{2}-\frac{g(0)}{4 \delta_{2}}\left(g^{\prime} \circ \nabla u\right)(t) .
\end{aligned}
$$

Exploiting Young's inequality, (2.1), Lemma 2.1, (2.3) and (3.4) to deal with the sixth term

$$
\begin{aligned}
& \left.\left|\frac{1}{\rho+1} \int_{\Omega}\right| u_{t}\right|^{\rho} u_{t} \int_{0}^{t} g^{\prime}(t-s)(u(t)-u(s)) \mathrm{d} s \mathrm{~d} x \mid \\
\leq & \frac{1}{\rho+1}\left(\delta_{2}\left\|u_{t}\right\|_{2(\rho+1)}^{2(\rho+1)}+\frac{1}{4 \delta_{2}} \int_{\Omega}\left(\int_{0}^{t} g^{\prime}(t-s)(u(t)-u(s)) \mathrm{d} s\right)^{2} \mathrm{~d} x\right) \\
\leq & \frac{1}{\rho+1}\left(\delta_{2}\left\|u_{t}\right\|_{2(\rho+1)}^{2(\rho+1)}-\frac{g(0) c_{s}^{2}}{4 \delta_{2}} \int_{\Omega} \int_{0}^{t} g^{\prime}(t-s)|\nabla u(t)-\nabla u(s)|^{2} \mathrm{~d} s \mathrm{~d} x\right) \\
\leq & \frac{\delta_{2} c_{s}^{2(\rho+1)}}{\rho+1}(2 E(0))^{\rho}\left\|\nabla u_{t}\right\|_{2}^{2}-\frac{g(0) c_{s}^{2}}{4 \delta_{2}(\rho+1)}\left(g^{\prime} \circ \nabla u\right)(t) .
\end{aligned}
$$

A substitution of (3.20)-(3.25) into (3.19) leads to

$$
\begin{aligned}
\Psi^{\prime}(t) \leq \delta c_{8} \| & \nabla u \|_{2}^{2}+c_{9}(g \circ \nabla u)(t)-c_{10}\left(g^{\prime} \circ \nabla u\right)(t) \\
& +\left(c_{11}-\int_{0}^{t} g(s) \mathrm{d} s\right)\left\|\nabla u_{t}\right\|_{2}^{2}+\left|\mu_{2}\right| \delta_{2} \int_{\Omega} u_{t}^{2}(x, t-\tau(t)) \mathrm{d} x \\
& -\frac{1}{\rho+1}\left(\int_{0}^{t} g(s) \mathrm{d} s\right)\left\|u_{t}\right\|_{\rho+2}^{\rho+2},
\end{aligned}
$$

where $c_{8}=1+2(1-l)^{2}, c_{9}=\left(2 \delta+\frac{1}{2 \delta}+\frac{\mu_{1} c_{s}^{2}+\mu_{2} c_{s}^{2}}{4 \delta_{2}}\right)(1-l), c_{10}=\frac{g(0) c_{s}^{2}}{4 \delta_{2}(\rho+1)}+\frac{g(0)}{4 \delta_{2}}$, and $c_{11}=\delta_{2} \mu_{1} c_{s}^{2}+$ $\delta_{2}+\frac{\delta_{5} c_{s}^{2(\rho+1)}}{\rho+1}(2 E(0))^{\rho}$. Additionally, since $g$ is positive, continuous and $g(0)>0$, for any $t_{0}>0$, we have

$$
\int_{0}^{t} g(s) \mathrm{d} s \geq \int_{0}^{t_{0}} g(s) \mathrm{d} s=g_{0}, \quad \forall t \geq t_{0} .
$$

Hence, we conclude from (3.5), Lemma 3.1, (3.18), (3.26) and (3.27) that for any $t \geq t_{0}>0$,

$$
\begin{aligned}
& G^{\prime}(t)=M E^{\prime}(t)+\varepsilon \Phi^{\prime}(t)+\Psi^{\prime}(t) \\
& \leq\left(\frac{M}{2}-c_{10}\right)\left(g^{\prime} \circ \nabla u\right)(t)-\frac{g_{0}-\varepsilon}{\rho+1}\left\|u_{t}\right\|_{\rho+2}^{\rho+2}+\left(\frac{\varepsilon(1-l)}{2 l}+c_{9}\right)(g \circ \nabla u)(t) \\
&-\left(\varepsilon\left(\frac{l}{2}-\delta_{1} c_{s}^{2}\left(\mu_{1}+\left|\mu_{2}\right|\right)\right)-\delta c_{8}\right)\|\nabla u\|_{2}^{2} \\
&-\left(g_{0}-\varepsilon\left(\frac{\mu_{1} c_{s}^{2}}{4 \delta_{1}}+1\right)-c_{11}\right)\left\|\nabla u_{t}\right\|_{2}^{2} \\
&-\left(c_{2} M-\frac{\varepsilon\left|\mu_{2}\right|}{4 \delta_{1}}-\left|\mu_{2}\right| \delta_{2}\right) \int_{\Omega} u_{t}^{2}(x, t-\tau(t)) \mathrm{d} x
\end{aligned}
$$




$$
-\frac{\lambda M \zeta}{2} \int_{t-\tau(t)}^{t} \int_{\Omega} e^{-\lambda(t-s)} u_{t}^{2}(x, s) \mathrm{d} x \mathrm{~d} s .
$$

At this point, we choose $\delta_{1}$ such that

$$
\delta_{1} c_{s}^{2}\left(\mu_{1}+\left|\mu_{2}\right|\right) \leq \frac{l}{4}
$$

and select $\delta$ to satisfy

$$
\delta \leq \frac{\varepsilon l}{8 c_{8}}
$$

Then, let $\delta_{2}$ be small so that

$$
c_{11}=\delta_{2}\left(\mu_{1} c_{s}^{2}+1+\frac{c_{s}^{2(\rho+1)}}{\rho+1}(2 E(0))^{\rho}\right) \leq \frac{g_{0}}{2},
$$

After that, we choose $\varepsilon$ so that

$$
\varepsilon<\min \left\{\frac{\frac{g_{0}}{4}}{\frac{\mu_{1} c_{s}^{2}}{4 \delta_{1}}+1}, g_{0}\right\} .
$$

Finally, we pick $M$ sufficiently large such that

$$
M>\max \left\{2 c_{10}, \frac{\varepsilon \frac{\left|\mu_{2}\right|}{4 \delta_{1}}+\left|\mu_{2}\right| \delta_{2}}{c_{2}}\right\} .
$$

Consequently, there exist two positive constants $\lambda_{1}$ and $\lambda_{2}$ satisfying

$$
G^{\prime}(t) \leq-\lambda_{1} E(t)+\lambda_{2}(g \circ \nabla u)(t), \quad \text { for all } t \geq t_{0} .
$$

Multiplying (3.28) by $\xi(t)$, we have

$$
\xi(t) G^{\prime}(t) \leq-\lambda_{1} \xi(t) E(t)+\lambda_{2} \xi(t)(g \circ \nabla u)(t) .
$$

Then, employing the assumption $g^{\prime}(t) \leq-\xi(t) g(t)$ by (2.3) and using the fact that $-\left(g^{\prime} \circ \nabla u\right)(t) \leq$ $-2 E^{\prime}(t)$ by Lemma 3.1, we get

$$
\begin{aligned}
\xi(t) G^{\prime}(t) & \leq-\lambda_{1} \xi(t) E(t)-\lambda_{2}\left(g^{\prime} \circ \nabla u\right)(t) \\
& \leq-\lambda_{1} \xi(t) E(t)-2 \lambda_{2} E^{\prime}(t), \quad \text { for all } t \geq t_{0} .
\end{aligned}
$$

Next, we define

$$
F(t)=\xi(t) G(t)+2 \lambda_{2} E(t),
$$


which is equivalent to $E(t)$ by Lemma 3.3. Then, using (3.29) and the assumption $\xi^{\prime}(t) \leq 0$, $\forall t \geq 0$ by (A1), we obtain

$$
\begin{aligned}
F^{\prime}(t) & \leq \xi^{\prime}(t) G(t)-\lambda_{1} \xi(t) E(t) \\
& \leq-\lambda_{1} \xi(t) E(t) \leq-\lambda_{3} \xi(t) F(t), \quad \forall t \geq t_{0}
\end{aligned}
$$

for some positive constant $\lambda_{3}$. An integration of (3.30) over $\left(t_{0}, t\right)$ gives

$$
F(t) \leq F(0) e^{-\lambda_{3} \int_{t_{0}}^{t} \xi(s) \mathrm{d} s}, \quad \forall t \geq t_{0}
$$

Therefore, the equivalent relation between $F(t)$ and $E(t)$ yields

$$
E(t) \leq K e^{-\alpha \int_{t_{0}}^{t} \xi(s) \mathrm{d} s}, \quad \forall t \geq t_{0},
$$

where $\alpha$ and $K$ are some positive constants. This completes the proof.

Remark 3.5. We illustrate the energy decay rate given by Theorem 3.4 through the following examples which are introduced in $[5,25]$.

(1) If $g(t)=\frac{a}{(1+t)^{v}}$, for $a>0$ and $v>1$, then $\xi(t)=\frac{v}{1+t}$ satisfies the condition (2.3). Thus (3.31) gives the estimate

$$
E(t) \leq K(1+t)^{-\alpha} \text {. }
$$

(2) If $g(t)=a e^{-b(1+t)^{v}}$, for $a, b>0$ and $0<v \leq 1$, then $\xi(t)=b v(1+t)^{v-1}$ satisfies the condition (2.3). Thus (3.31) gives the estimate

$$
E(t) \leq K e^{-\alpha(1+t)^{v}}
$$

(3) If $g(t)=a e^{-b \ln v(1+t)}$, for $a, b>0$ and $v>1$, then $\xi(t)=\frac{b v \ln ^{v-1}(1+t)}{1+t}$ satisfies the condition (2.3). Thus (3.31) gives the estimate

$$
E(t) \leq K e^{-\alpha \ln ^{v}(1+t)}
$$

(4) If $g(t)=\frac{a}{(1+t) \ln ^{v}(1+t)}$, for $a>0$ and $v>1$, then $\xi(t)=\frac{\ln (1+t)+v}{(1+t) \ln ^{v}(1+t)}$ satisfies the condition (2.3). Thus (3.31) gives the estimate

$$
E(t) \leq K\left((1+t) \ln ^{v}(1+t)\right)^{-\alpha} .
$$

\section{References}

[1] Abdallah C., Dorato P., Benitez-Read J. and Byrne R., Delayed positive feedback can stabilize oscillatory system, ACC. San Francisco (1993), 3106-3107.

[2] Datko R., Not all feedback stabilized hyperbolic systems are robust with respect to small time delays in their feedbacks, SIAM J. Control Optim., 26 (1988), 697-713. 
[3] Datko R., Two examples of ill-posedness with respect to time delays revisited, IEEE Trans. Automatic Control, 42 (1997), 511-515.

[4] Datko R., Lagnese, J. and Polis, M. P., An example on the effect of time delays in boundary feedback stabilization of wave equations. SIAM J. Control Optim., 24 (1) (1986), 152-156.

[5] Kirane M., Belkacem S. H., Existence and asymptotic stability of a viscoelastic wave equation with a delay, Z. Angew. Math. Phys., DOI 10.1007/s00033-011-0145-0.

[6] Xu G. Q., Yung S. P. and Li L. K., Stabilization of the wave system with input delay in the boundary control. ESAIM: Control Optim. Calc. Var., 12 (2006), 770-785.

[7] Nicaise S., Pignotti C., Stability and instability results of the wave equation with a delay term in the boundary or internal feedbacks. SIAM J. Control Optim., 45 (5) (2006), 1561-1585.

[8] Nicaise S., Pignotti C., Stabilization of the wave equation with boundary or internal distributed delay. Diff. Int. Equs., 21 (9-10) (2008), 935-958.

[9] Nicaise S., Valein J., Stabilization of the wave equation on 1-d networks with a delay term in the nodal feedbacks. Netw. Heterog. Media., 2 (3) (2007), 425-479.

[10] Nicaise S., Valein J. and Fridman E., Stabilization of the heat and the wave equations with boundary time-varying delays. DCDS-S., 2 (3) (2009), 559-581.

[11] Nicaise S., Pignotti C. and Valein J., Exponential stability of the wave equation with boundary time-varying delay, DCDS-S., 4 (3) (2011), 693-722.

[12] Suh I. H., Bien Z., Use of time delay action in the controller design. IEEE Trans. Automat. Control., 25 (1980), 600-603.

[13] Lasiecka I., Triggiani R. and Yao P. F., Inverse/observability estimates for second-order hyperbolic equations with variable coefficients, Journal of Mathematical Analysis and Applications, 235 (1999), 13-57.

[14] Liu W. J., General decay of the solution for a viscoelastic wave equation with a time-varing delay term in the unternal feedback, J. Math. Phys., 54 (2013), 043504.

[15] Cavalcanti M. M., Domingos Cavalcanti V N. and Ferreira J., Existence and uniform decay of nonlinear viscoelastic equation with strong damping, Mathematical Methods in Applied Sciences, 24 (2001), 1043-1053.

[16] Messaoudi S. A. and Tatar N-e., Global existence and asymptotic behavior for a nonlinear viscoelastic problem, Mathematical Science Research Journal, 7 (4) (2003), 136-149.

[17] Messaoudi S. A., Tatar N-e., Global existence and uniform stability of solutions for a quasilinear viscoelastic problem, Mathematical Methods in Applied Sciences, 30 (2007), 665-680.

[18] Han Xiaosen, Wang Mingxin, General decay of energy for a viscoelastic equation with nonlinear damping, Mathematical Methods in Applied Sciences, 32 (2009), 346-358.

[19] Han Xiaosen, Wang Mingxin, Global existence and uniform decay for a nonlinear viscoelastic equation with damping, Nonlinear Anal., Theory, Methods \& Applications, 70 (9) (2009), 3090-3098.

[20] Liu W. J., General decay rate estimates for a viscoelastic equation with weakly nonlinear time-dependent dissipation and source terms, J. Math. Phys., 50 (2009), 113506.

[21] Messaoudi S. A., Tatar N-e., Global existence and asymptotic behavior for a nonlinear viscoelastic problem, Mathematical Science Research Journal, 7 (4) (2003), 136-149.

[22] Messaoudi S. A., Tatar N-e., Exponential decay for a quasilinear viscoelastic equation, Math. Nachr., 282 (10) (2009), 1443-1450.

[23] Wu S. T., Asymptotic behavior for a viscoelastic wave equation with a delay term, Taiwanese Journal of Mathematics, 17 (3) (2013), 765-784.

[24] Wu S. T., General decay of solutions for a viscoelastic equation with nonlinear damping and 
source terms, Acta Mathematica Scientia, 31(B) (4) (2011), 1436-1448.

[25] Messaoudi S. A., General decay of solutions of a viscoelastic equation, Journal of Mathematical Analysis and Applications, 341 (2008), 1457-1467. 